\title{
Analysis of Parametric Resonances in Comb-Driven Microscanners
}

\author{
Caglar Ataman, Ozgur Kaya, Hakan Urey \\ Optical Microsystems Laboratory, Department of Electrical Engineering, Koc University \\ Rumeli Feneri Yolu, 34450, Sariyer, Istanbul, TURKEY \\ Phone: +90-212-338-1474, E-mail: cataman@ku.edu.tr, hurey@ku.edu.tr
}

\begin{abstract}
Dynamic behavior of a comb-driven torsional microscanner is governed by a nonlinear parametric differential equation. Theoretically, such systems have multiple resonances located near the integer fractions of twice the mechanical resonance frequency. The number of observable parametric resonances strongly depends on the damping of the system, whereas the stable and unstable operating regions are determined by drive-voltage and drive-frequency. In atmospheric pressure, only first few of these parametric resonances are observable within the operation voltage range of the devices. This paper explores the effect of damping on the various characteristics of parametric resonances and some unusual scanner behavior rarely seen in mechanical structures. A numerical and an analytical model for comb-driven microscanners are presented. Frequency responses of various devices are experimentally measured inside a vacuum chamber at different ambient pressures ranging from atmospheric pressure to 30 mTorr. Experimental results are compared with analytical and simulation results.
\end{abstract}

Keywords: Comb-drive actuation, microscanner, subharmonic oscillation, parametric resonance, damping, quality factor

\section{INTRODUCTION}

Electrostatic comb-drive actuation is a common actuation mechanism in microelectromechanical systems (MEMS). Comb-drives can be actuated in in-plane or out-of plane modes, and operation mode of the actuator is the fundamental factor that determines the dynamic behavior of the associated device. In-plane mode comb-actuators are widely utilized in various devices and systems such as tunable capacitors ${ }^{1}$, mechanical resonators ${ }^{2}$, optical switches ${ }^{3}$, and mechanical filters for RF and other applications., ${ }^{4} 5$ In this mode of operation, the force induced by the actuator is independent of the displacement of the combs, and the actuator exhibits a simple harmonic oscillatory (SHO) behavior. As all other second order harmonic oscillator, in-plane mode comb actuators have one resonance, corresponding to the mechanical resonance of the system. On the other hand, the force in out-of-plane actuator is both time and displacement dependent. This relatively complicated nature of the forcing function leads to the phenomenon of parametric resonance. Parametric systems are seen in different areas of science and engineering, such as photonic crystals ${ }^{6}$, optical cavities ${ }^{7}$, plate vibrations ${ }^{8}$, and quantum devices. ${ }^{9}$ A parametric oscillator is described with a second order differential equation with time-dependant coefficients. In parametric systems, linear relation between the drive signals and the output of the system ceases to exist and the system exhibits subharmonic oscillations and hysterical frequency response.

We have previously proved that a comb-driven torsional microscanner is a typical parametric oscillator and presented experimental results, numerical model, and analytical solutions for the primary resonance (fundamental parametric resonance). ${ }^{10}$ This paper explores the effect of damping and drive voltage on the number and width of the stability curves of the microscanners. We applied "method of strained parameters" to obtain stability regions in the analytical solution, and we applied "Floquet Theory" to obtain our approximate transient analytical solutions. Our analytical solutions successfully predict stability regions and other characteristics of primary and higher order resonances. In section II, we give a brief introduction to the microscanner structure. Section III includes the analytical analysis of the stability regions and steady state solutions for the parametric resonance. Experimental frequency response and stability data are also given in section III. Comparisons of this data with analytical and numerical results are also included in this section. In the last part, we included conclusions and a brief discussion on the significance of this work. 


\section{THE DEVICE}

\subsection{Device Layout}

The device that is investigated in this work is a 1D, $30 \mu \mathrm{m}$ thick single-crystalline silicon comb-actuated microscanner. The structure consists of a movable, aluminum covered $2 \mathrm{~mm}$ x $2 \mathrm{~mm}$ aluminum mirror, a fixed frame and two flexure beams that bind the mirror to the frame. The flexures are $550 \mu \mathrm{m}$ in length, $30 \mu \mathrm{m}$ in width, $20 \mu \mathrm{m}$ in thickness, and they act as torsional springs that pull the mirror back to its rest position. The mirror and the frame are electrically isolated via the isolation trenches. Therefore, different potentials can be applied to the complementary comb fingers. These fingers are placed on sidewalls of the mirror. The devices were fabricated by Fraunhofer Institute for Photonic Microsystems (IPMS), in Dresden-Germany. More information on the design and fabrication of these devices can be found in the related works by Fraunhofer IPMS ${ }^{11,12,13}$. Figure 1shows the general micromirror layout.

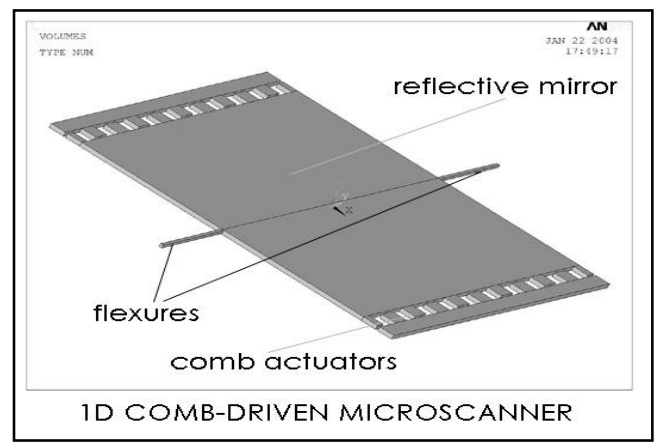

Figure 1 - General layout of the microscanner

\subsection{Actuation Mechanism}

Torsional movement of these micromirrors is induced by the numerous comb shaped fingers on the edge of the device. These fingers greatly enhance the overlap capacitance between the opposing surfaces and lower the operation voltage of an electrostatic actuator significantly. Figure 2 shows the comb fingers on the microscanner and a first order approximation to capacitance-change - tilt-angle characteristics of the comb actuator.

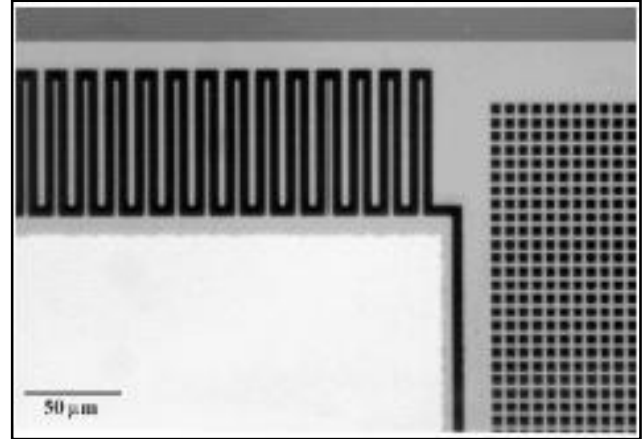

(a)

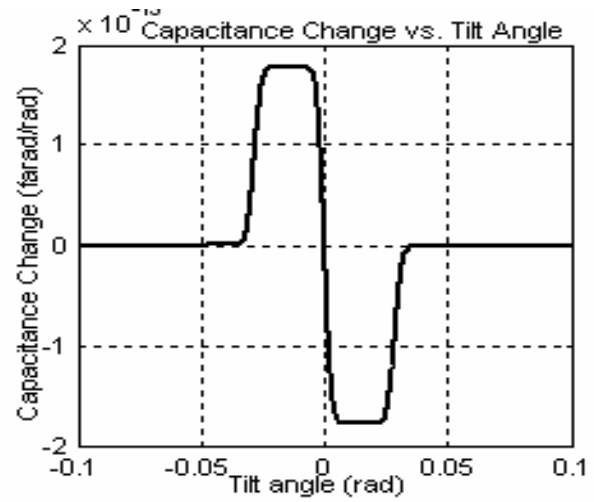

(b)

Figure 2 - (a) The comb fingers on the edge of the microscanner mirror. (b) Torque vs. tilt angle characteristic

The amount of torque produced by the comb-drive actuators is given by the expression,

$$
M(\theta)=2 N \frac{1}{2} \frac{d C}{d \theta} V(t)^{2}
$$

where, $N$ is the number of comb fingers. The nonlinear relation between the rate of change of capacitance and the tilt angle is showed in Figure 2(b). In electrostatic actuators, the amount of torque produced is proportional to the square of 
the input signal. Therefore, when a time-varying input is applied to the system, the torque function is both time and displacement dependant. We have previously shown that this nature of the torque function leads to parametric oscillations, and comb-drive microscanners exhibit the typical characteristics of parametric systems. Figure 3 shows the frequency response of the device within the first parametric resonance.

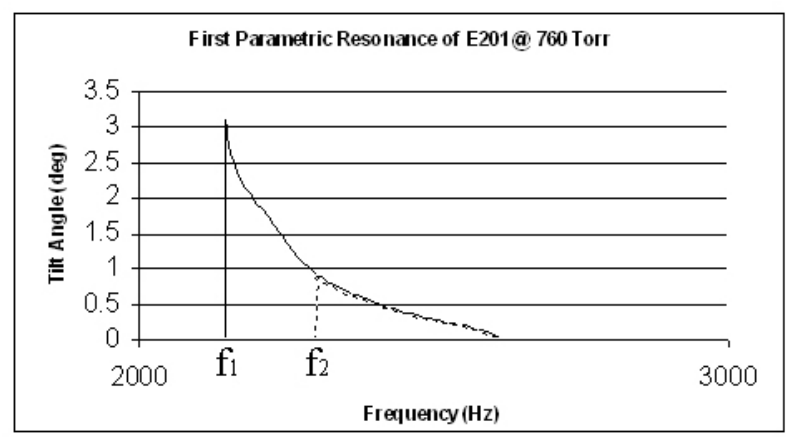

Figure 3 - Frequency response curve of the device in the fundamental parametric resonance. The peak amplitude corresponds to $\mathrm{f} \approx 2 f_{0}$, where $f_{0}$ is the mechanical resonance frequency of the system.

The shape of the response curve is typical for all similar devices. Frequency Response of the torsional comb-driven microscanners is a nonlinear function exhibiting subharmonic oscillations and hysterical behavior. The path traced on the $\theta-f$ curve when frequency is increased quasi-statically from a lower frequency is different than the case, in which the frequency is swept down from a higher frequency. The interval between the two jump frequencies $f_{1}$ and $f_{2}$ is the unstable region of the response curve. Oscillations in this region can only be observed if the external frequency is quasistatically swept down to this region from a higher initial value. At the critical frequencies, the jump in the oscillation amplitude is extremely abrupt - sharper than $10^{-3} \mathrm{~Hz}$, which is the maximum resolution of our measurements. It is uncommon for a macroscopic parametric system to have multiple observable parametric resonances. But we are able observe more than one resonance even in the atmospheric pressure. In vacuum, the number of observable resonances is much higher. The shape of the other orders of resonance is still the same, but they are located around $2 f_{0} / n, n=1,2, \ldots$. In the first parametric resonance, the oscillation frequency is half the driving signal frequency. This phenomenon is called subharmonic oscillation. Subharmonic oscillations in comb-driven microscanners are explored in our earlier work. ${ }^{10}$

\section{THEORY, EXPERIMENTS AND SIMULATIONS}

General equation of motion for a microscanner can be written as follows ${ }^{18}$ :

$$
I_{m} \frac{d^{2} \theta}{d t^{2}}+b \frac{d \theta}{d t}+K_{f} \theta=M(\theta)
$$

where,

$$
\begin{aligned}
& I_{m}: \text { Mass moment of inertia; } \\
& b \text { : Damping constant; } \\
& K_{f} \text { : Torsional stiffness; } \\
& M(\theta) \text { : Torque function. }
\end{aligned}
$$

For very small tilt angles, the torque function of the comb actuators can be successfully approximated by a $3^{\text {rd }}$ order odd polynomial. With a square-rooted-sine input voltage $(V=A \sqrt{\cos (w t)+1})$, the torque expression is;

$$
M(\theta, t)=-\left(r_{3} \theta^{3}+r_{1} \theta\right) A^{2}(\cos (w t)+1)
$$

We can now rewrite Eq.2 as follows: 


$$
\frac{d^{2} \theta}{d \tau^{2}}+\alpha \frac{d \theta}{d \tau}+(\beta+2 \delta \cos (2 \tau)) \theta+\left(\delta_{3}+\delta_{3} \cos (2 \tau)\right) \theta^{3}=0
$$

where,

$$
\tau=\frac{w t}{2}, \quad \alpha=\frac{2 b}{w I_{m}}, \quad \beta=4 \frac{\left(r_{1} A^{2}+K_{f}\right)}{w^{2} I_{m}}, \quad \delta=\frac{2 r_{1} A^{2}}{w^{2} I_{m}}, \quad \delta_{3}=4 \frac{r_{3} A^{2}}{w^{2} I_{m}}
$$

In this new representation $\tau$ stands for the new time variable, which is the actual time scaled by $w / 2$. Eq.4 is a nonlinear homogeneous Mathieu equation and there exists two linearly independent solutions for this equation. We have previously presented approximate analytical expressions for the behavior of this system near first parametric resonance. ${ }^{10}$ A more general insight for the transient behavior of the system may be gained by using Floquet theory. In order to utilize this theory, we need to express Eq.4 in state space representations, as follows:

$$
\begin{aligned}
& \dot{x}_{1}=x_{2} \\
& \dot{x}_{2}=-\alpha x_{2}-(\beta+2 \delta \cos (2 \tau)) x_{1}-\left(\delta_{3}+\delta_{3} \cos (2 \tau)\right) x_{1}{ }^{3}
\end{aligned}
$$

An analytical solution can be obtained when the cubic nonlinearity and the damping terms are ignored. We can now, rewrite this system of equations in matrix form as: ${ }^{16}$

$$
\left[\begin{array}{l}
\dot{x}_{1} \\
\dot{x}_{2}
\end{array}\right]=\left[\begin{array}{cc}
0 & 1 \\
-f(\tau) & 0
\end{array}\right]\left[\begin{array}{l}
x_{1} \\
x_{2}
\end{array}\right]
$$

Where, $f(t)=\beta+2 \delta \cos (\tau)$. Linear system theory suggests that this system of equations has two linearly independent solutions. Let $X(t)$ be a fundamental solution matrix, whose columns are two linearly independent solutions of Eq.6, and satisfy the initial conditions $X(0)=I$, where $I$ is the identity matrix. Floquet theory suggests that the characteristic matrix $C$ of Eq.6 is given by the relation, ${ }^{14}$

$$
C=X(T)=\left[\begin{array}{ll}
x_{1}(T) & \dot{x}_{1}(T) \\
x_{2}(T) & \dot{x}_{2}(T)
\end{array}\right]
$$

Where, $T$ is the period of $f(\tau)$, and equals to $2 \pi$ for the Mathieu equation. The transient solutions and the stability of the system in Eq. 3 can be determined using the characteristics matrix. Let $\theta_{1,2}(\mathrm{t})$ denote the transient solutions of the system of interest; then they are given by the relation. ${ }^{14}$

$$
\begin{aligned}
& \theta_{1}(\tau)=e^{\gamma_{1} \tau} \phi_{1}(\tau) \\
& \theta_{2}(\tau)=e^{\gamma_{21} \tau} \phi_{2}(\tau)
\end{aligned}
$$

where, $y_{i}$ are called the characteristic exponents of the equation. Above expression is the general form of the solution for linear Mathieu equation. In torsional comb microscanner case, the periodic functions of $\phi_{i}(\tau)$ are linear combinations of sinusoidal functions of period $\pi$ and $2 \pi \cdot y_{i}$ are given by the expression,

$$
\gamma_{i}=\frac{1}{T} \ln \left(\lambda_{i}\right)
$$

Where, $\lambda_{i}$ are the eigenvectors of the characteristic matrix $C$. For a linear Mathieu equation, the product of these eigenvectors is unity. Depending on the values of these eigenvectors, steady state and stability characteristics of the system can be determined. If these eigenvectors are complex conjugates, they have unity modulus and therefore they lie on the unit circle. In this case, the steady state output is bounded and the system is stable. However, the amplitude of the output has a damped oscillatory behavior, due to Eq.8. Figure 4(a) shows the experimental data for such a behavior. The 
system is within first parametric resonance range, but is far from its resonance peak. The exponential growth and damped oscillations in the output amplitude can be clearly seen from the data. When one of the eigenvectors of the characteristic matrix has amplitude greater than unity, one of the fundamental solutions die out in time, however the amplitude of the other solution grows exponentially. If there is no damping in the system, this growth in the output amplitude is unbounded, and the system becomes unstable. However, both damping and the cubic nonlinearity, -the effects that we both ignored in deriving Eq.8- have a stabilizing effect on the system, and they limit the oscillation amplitude of the unstable system. When $f_{e x c}=f_{l}$ or $f_{e x c}=f_{l}$, the eigenvectors of the characteristic matrix are either -1 or 1 , and the oscillatory behavior in the transient response diminishes. Such a characteristic can be observed in Figure4b. Unlike linear systems, exponential terms has positive growth coefficient, and the amplitude grows until it reaches upper bound set by damping and the cubic nonlinearity. Then suddenly, oscillation amplitude stops growing.

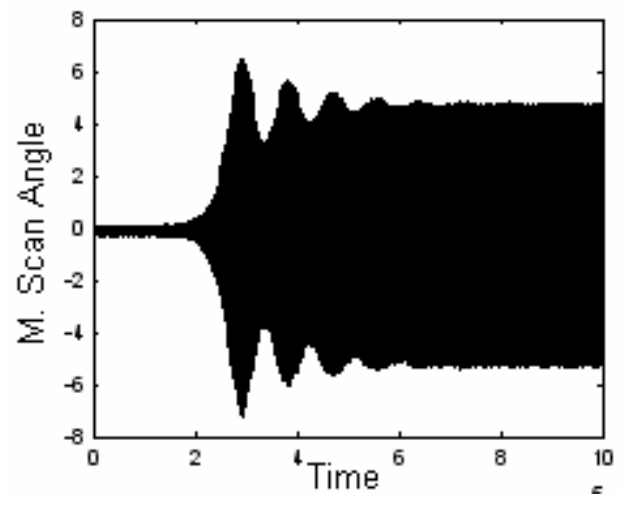

(a)

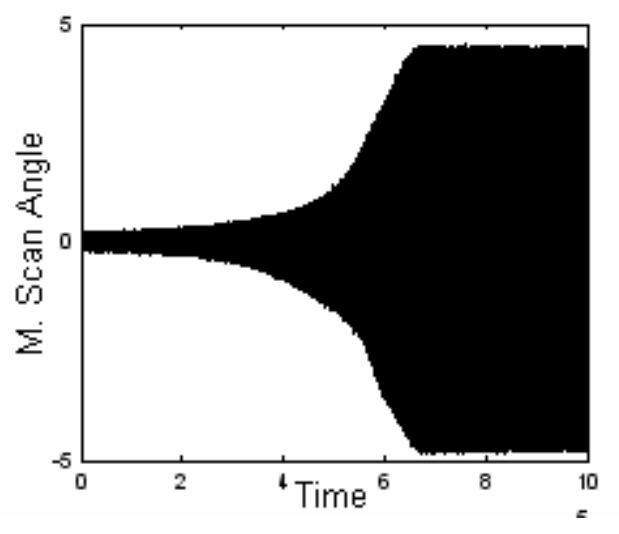

(b)

Figure 4 - Transient response of the device to various frequencies. (a) $f_{\text {ext }}>f_{2}$ (stable region);

(b) $f_{e x t}=\mathbf{f}_{2}$ (boundary of the unstable region).

\subsection{Stability Tongues}

According to the Floquet theory, the solutions of Eq.4 on the stability transition curves have periods of $\pi$ or $2 \pi$, and these curves correspond to the positive or negative unity eigenvectors of the characteristic matrix. These stability curves form a tongue-like shape in the drive-voltage - drive-frequency space, and therefore called "stability tongues". Due to the time scaling of Eq.4, a solution which is of period $\pi$ in the new time scale correspond to an oscillation of period $T$ in the actual time. Similarly, the $2 \pi$ periodic solution of Eq. 4 is actually an oscillation of period $2 T$. This $2 T$ periodic solution is half the frequency of the drive signal and corresponds to the subharmonic oscillation in the $1^{\text {st }}$ order parametric resonance. Based on the result of Floquet theory that on boundaries of the stability tongues the solution of Eq. 4 is either $\pi$ or $2 \pi$ periodic, we utilized the method of strained parameters, in order to find analytical expressions for determining these tongues. In this method the characteristic exponents are chosen so that the periods of the possible solutions of Eq. 4 are $\pi$ or $2 \pi$. Then, the required parameters are determined in such a way that this assumption holds. With the effect of small viscous damping, which is the case in comb-driven microscanners within small oscillation amplitudes, the transition curves separating the stable and unstable regions are given by ${ }^{14}$ :

$$
\beta=n^{2} \pm \frac{1}{2}\left[\left({a_{1 n}}^{2}+b_{1 n}{ }^{2}\right) \delta^{2}-16 \alpha^{2} n^{2}\right]^{\frac{1}{2}}+O(n), \quad \mathrm{n}=1,2,3, \ldots
$$

Where, $a_{1 n}$ and $b_{1 n}$ are the Fourier series coefficients of $f(t)$, and $\mathrm{O}(n)$ stands for higher order terms. For the first two orders of parametric resonances, the expressions for the associated stability tongues can be found as: ${ }^{15}$

$$
\begin{aligned}
& \beta=1 \pm\left(\delta^{2}-4 \alpha^{2}\right)^{\frac{1}{2}}-\frac{1}{8} \delta^{2}+O(n) \\
& \beta=4+\frac{1}{6} \delta^{2} \pm\left(\frac{1}{16} \delta^{2}-16 \alpha^{2}\right)^{\frac{1}{2}}+O(n)
\end{aligned}
$$


Figure 5 shows the experimental results and analytical approximations for the stability tongues for the observable parametric resonances at two different ambient pressure levels. The tongues are mapped in to drive-voltage - drivefrequency plane using the relations in Eq.4.

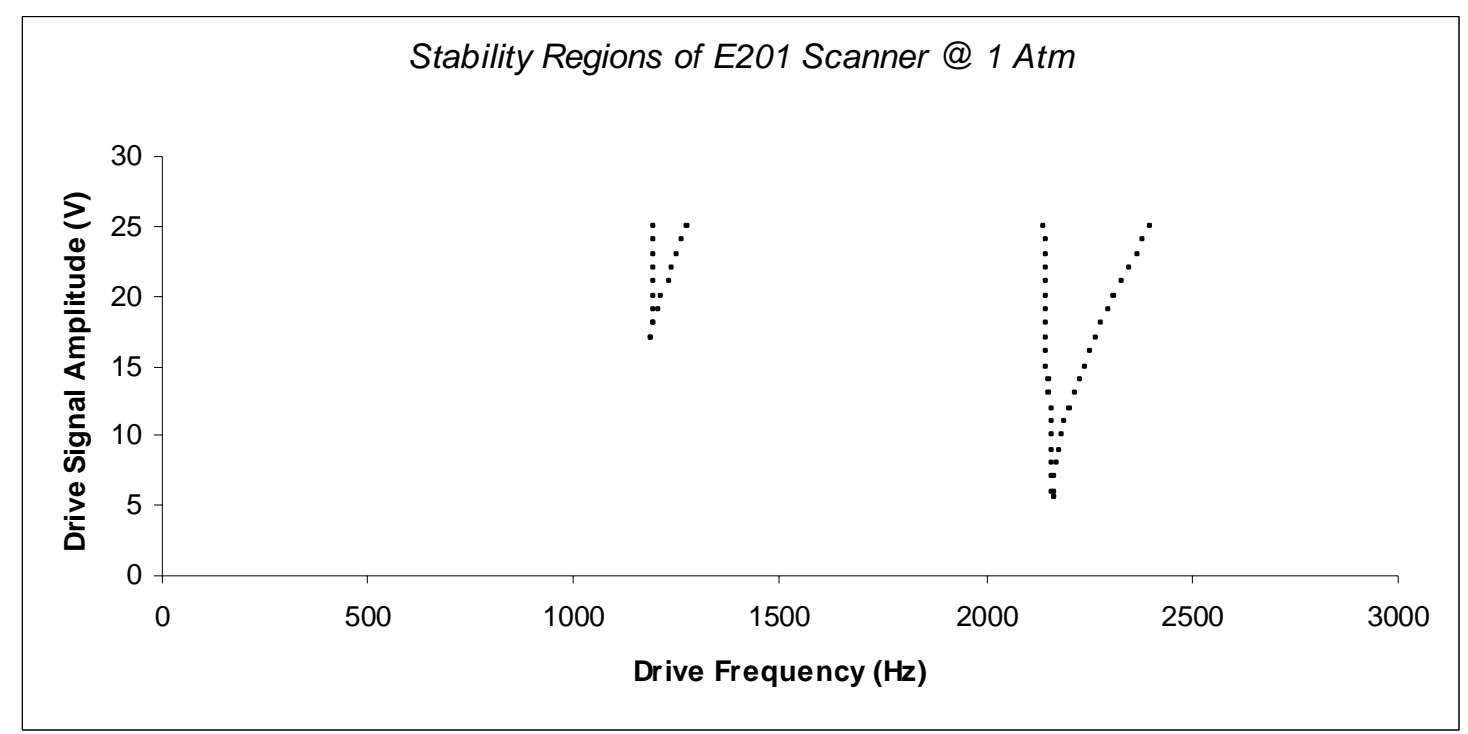

(a)

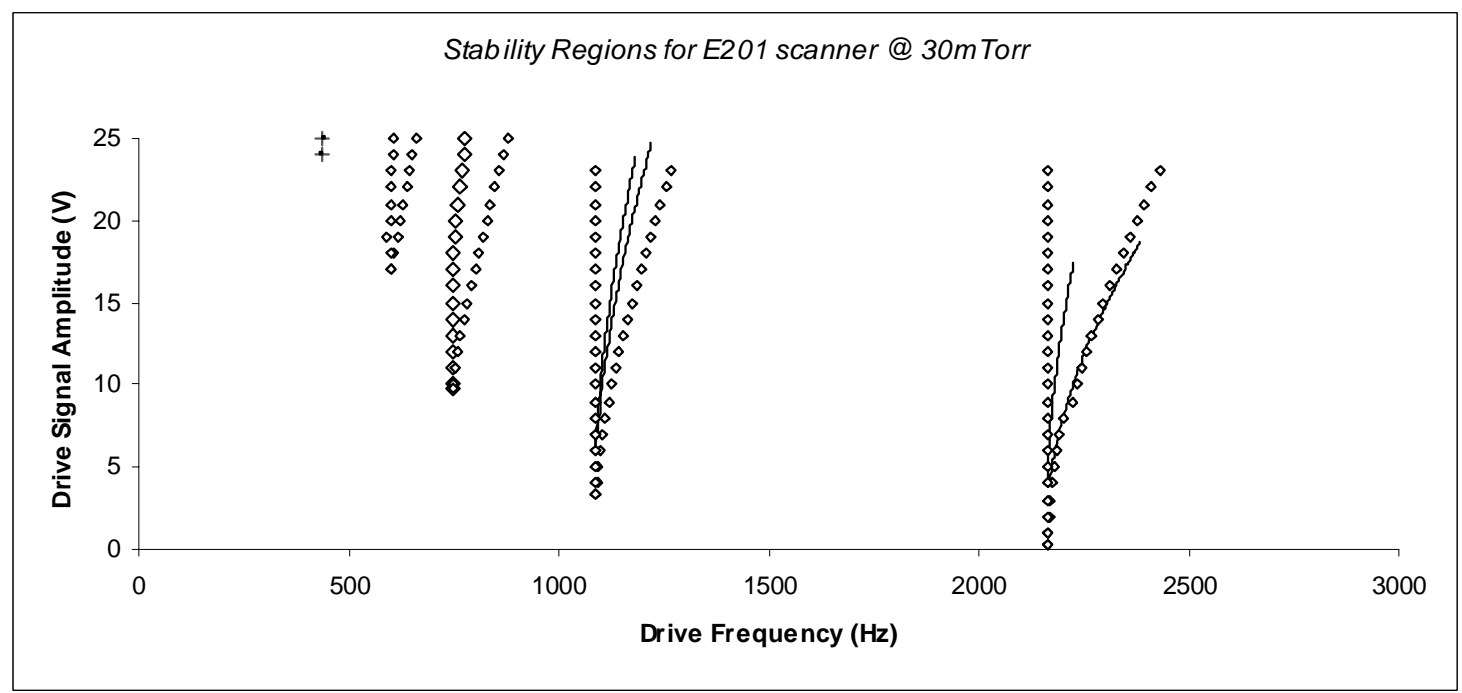

(b)

Figure 5 - Stability tongues for the device (a) At atmospheric pressure; (b) At an ambient pressure of 30 mTorr. Dotted lines show experimental results and solid lines in (b) show the stability tongues evaluated using the analytical formulas.

Figure 5(a) shows the stability tongues of the microscanner in the atmospheric pressure. There is a significant viscous air damping between the comb fingers in the atmospheric pressure (we have measured a Q factor of 22), so that there are only two observable parametric resonances within the operation voltage of the device. Additional orders or resonances would be observed with higher drive voltages, but we avoided such high voltages in order to prevent device failure. The stability tongues of the microscanner in $30 \mathrm{mT}$ orr ambient pressure can be seen in Figure $5 \mathrm{~b}$. Voltage and frequency span of the two plots are identical. In low pressure, air damping is much less and therefore the device has a much higher quality factor $(\sim 1250)$. As seen in the Figure 5(b), there are 5 observable parametric resonances and the width of the stability tongues are increased, and their tips came much closer to the $V=0$ axis. In Figure $5 b$, solid lines represent the 
results of the analytical expressions of the stability tongues. The analytical results are in good agreement with the experimental data. However, as the input voltage increases, the deviation of the analytical approximation from the experiments increases. This is due to the fact that for higher voltages, the small driving signal and small tilt angle approximations are becoming invalid and therefore our perturbation analysis ceases to be accurate. Another reason of the difference between the experimental and analytical results is the cubic nonlinearity in Eq.3. As the driving voltage increases, this cubic nonlinearity term also grows and it prevents the left hand of the stability curve to bend rightward.

\subsection{Frequency Response}

Damping of system also has a significant effect on the frequency response of the device. Figure 5 compares the frequency response of the comb-driven microscanner at two different ambient pressures. The solid line represents the experimental data for backward frequency sweep, and the dotted line represents forward frequency sweep. The driving signal was a square-root sine wave with peak-to-peak value of $20 \mathrm{~V}$.

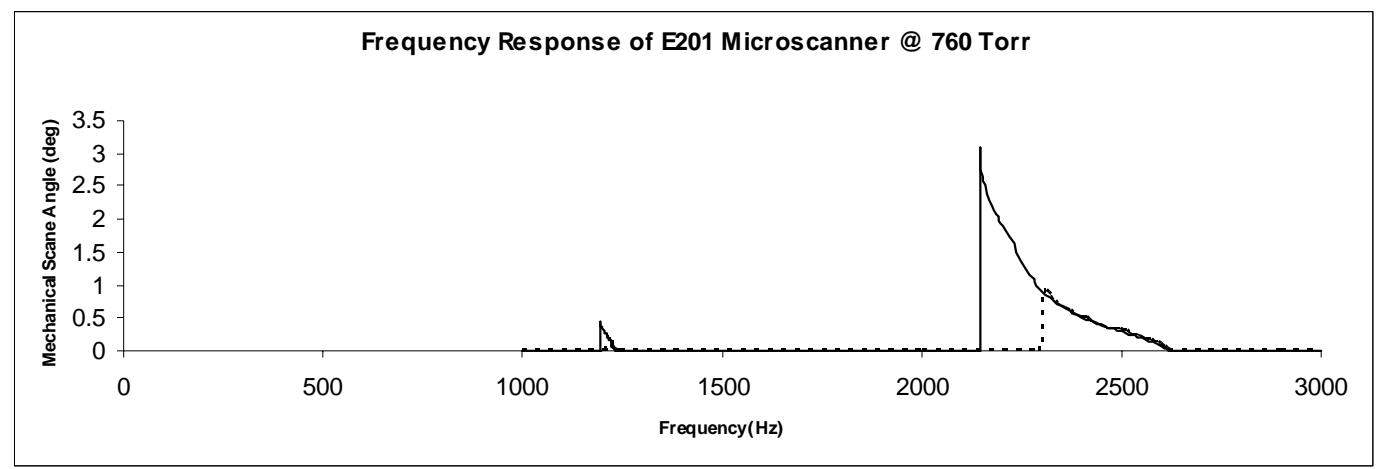

(a)

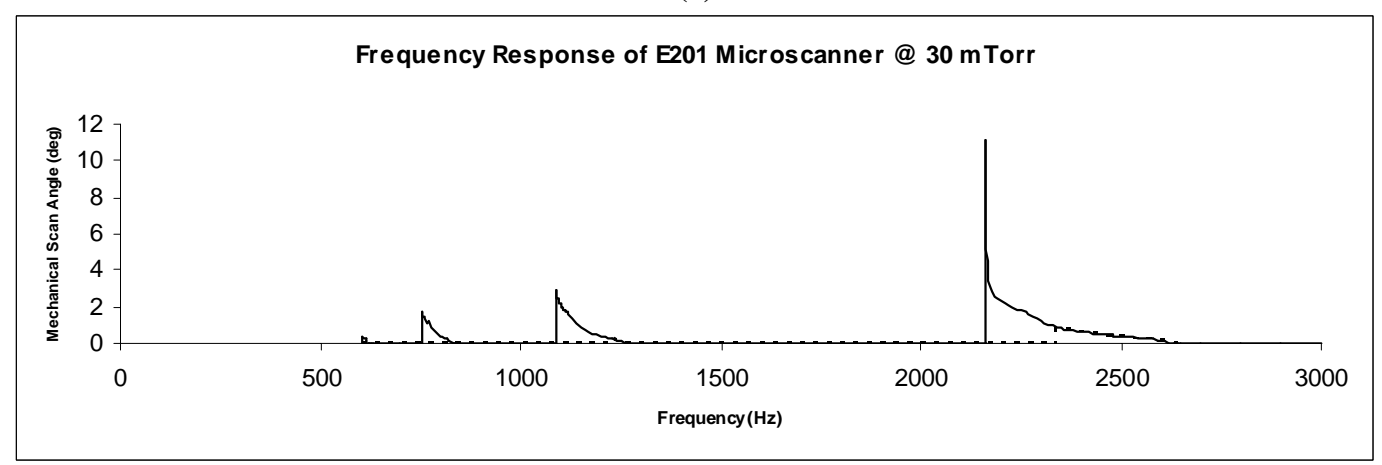

(b)

Figure 6. Frequency response of the microscanner; (a) at atmospheric pressure, (b) at 30 mTorr.

With this drive signal, we can observe two parametric resonances in the atmospheric pressure and four resonances in 30 mTorr. The amplitude of the oscillations is significantly increases in low pressure due to lower damping. Near the peak of the first parametric resonance, the oscillation amplitude reaches almost $12^{\circ}$. In atmospheric pressure, the maximum oscillation amplitude is around $3.5^{\circ}$. Another effect of the damping is the modification of the resonant frequency. ${ }^{14}$ When the pressure changed from ambient to 30 mTorr, resonance frequency of the device increased from $1068.9 \mathrm{~Hz}$ to $1081.7 \mathrm{~Hz}$. This shift is due to the frequency tuning effect of damping.

\subsection{Simulation Results}

Frequency and transient response of the devices were simulated using our numerical model. The simulations are done by numerically solving Eq.2 using the differential equation solvers in MATLAB ${ }^{\odot}$. The torque associated with the comb actuators is computed using a first order approximation of the total capacitance in the system (Figure 2(b)). We have presented the detailed modeling procedure elsewhere. ${ }^{10}$ Figure 7 is presents the results of some simulations run with this model. Figure 7(a) compares the experimental data and simulation results for the frequency response of the device at atmospheric pressure. The model accurately predicts the values of frequencies, where abrupt changes in the amplitude occur. There is a slight disagreement between the amplitudes of two data sets, which is due to the ignored fringing 
electric fields between the comb fingers. Figure 7(b) shows the result for a sample transient response simulation, which verifies the exponential increase and oscillatory decay in the output amplitude in this operation region observed in the experiments (see. Figure 4a). The numerical results show good agreement with the experimental data and verify the validity of the model.

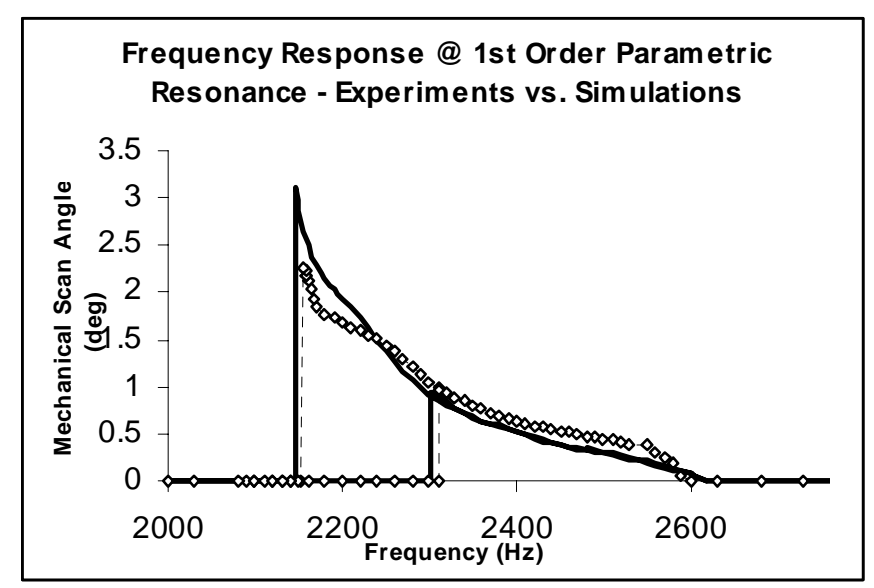

(a)

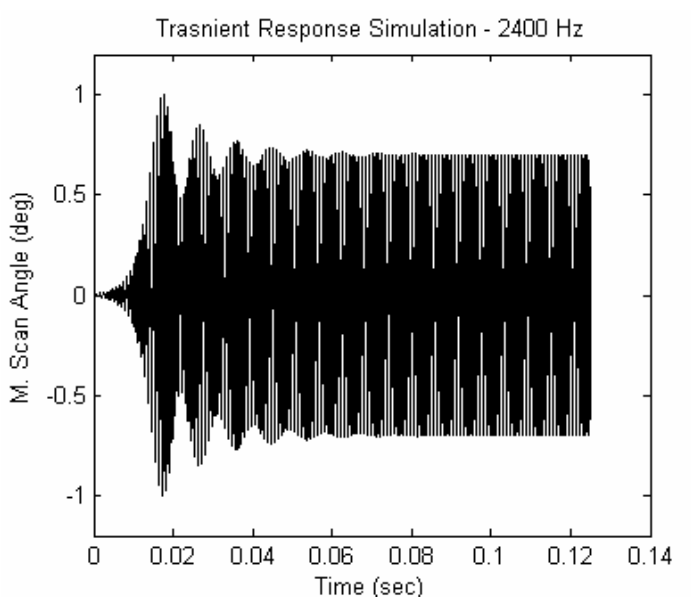

(b)

Figure 7 - Simulation results for; (a) frequency response at $1^{\text {st }}$ order parametric resonance, (b) sample transient response at stable region.

\subsection{Experimental Setup}

The microscanner structure does not employ an angular displacement feedback feature; therefore, we have used various optical characterization methods, in order to obtain desired data. Figure 8 shows a picture of our frequency response, transient response stability analysis measurements setup. Transient response measurements are done using a fast 2D-Position Sensing Detector (PSD) and a PC with data acquisition hardware. A laser beam is sent on the microscanner, and the scan waveform of the microscanner is recorded by reflecting the scan-line onto the PSD. Response time of the PSD is much faster than the scan frequency, therefore no deformation of the scan waveform is allowed. For the frequency response and stability measurements, we used a computer-controlled setup, based on a single photodetector. A similar method is used for the damping measurements. For the low-damping experiments, the device is placed inside a vacuum chamber with a transparent bell jar.

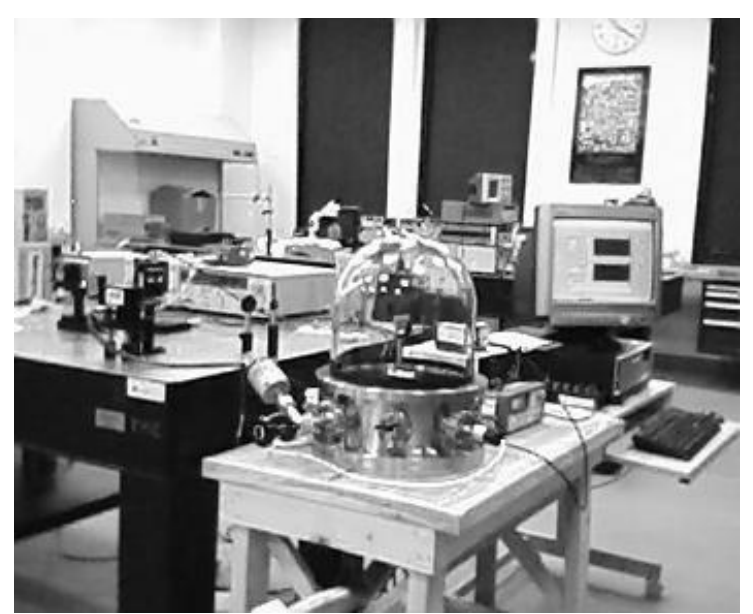

(a)

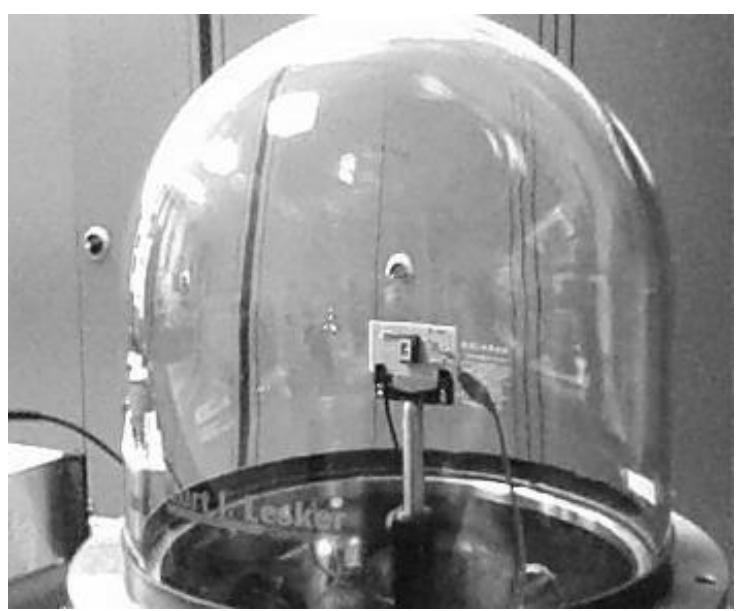

(b)

Figure 8 - Experiment setup. (a) General view of the setup. (b) Microscanner inside the vacuum chamber 


\section{DISCUSSION AND CONCLUSIONS}

In this work, we have presented analytical expressions describing the many fundamental characteristics of comb-driven microscanners. Having an analytical and numerical tool for predicting microscanner behavior is very valuable. In linear scanners, one avoids having other resonances in the system close to the primary resonance of the scanner and its harmonics. For the nonlinear parametric resonance scanner, one should also pay attention to the subharmonic frequencies in the system. Theory presented here is applied to various science and engineering problems, but to our knowledge, this is the first time that a detailed analysis of the stability, transient and frequency behavior of a parametric resonance device is presented. Together with the analytical expressions, we have presented experimental and simulation data and they confirm the analytical predictions very well. Our analytical model is valid within small damping and small oscillation angles, but it gives a good understanding of the stability and transient response. On the other hand, our numerical model is valid for both small and large scan angles, providing a very useful tool for scanner design and characterization.

\section{REFERENCES}

1. Z. Xiaoa, W. Penga, R.F. Wolffenbuttel, K.R. Farmera, "Micromachined variable capacitors with wide tuning range," Sensors and Actuators A, Vol.104, p.299-305 (2003).

2. C. T. C. Nguyen, "High-Q micromechanical oscillators and filters for communications," International Symposium on Circuits and Systems, Proc. IEEE, p.2825-2828 (1997).

3. J. D. Grade, K. Y. Yasumura, H. Jerman, "A DRIE comb-drive actuator with large, stable deflection range for use as an optical shutter, ” International Conference on Solid-State Sensors, Actuators and Microsystems, Proc. IEEE/EDS, Boston, Massachusetts, USA (2003).

4. L-J. Yanga, T-W. Huangb, P-Z. Changb, “CMOS microelectromechanical band-pass filters, ” Sensors and Actuators A, Vol. 90, p.148-152 (2001).

5. L. Lin, R. T. Howe, A. P. Pisano, "Micromechanical filters for signal processing," Journal of Microelectromechanical Systems, Vol. 7, No. 3, p.286-294 (1998).

6. V. V. Konotop, V. Kuzminak, "Parametric resonance of a defect mode in a 2D photonic crystal," Physical Review B, Vol. 64, p.1-7, (2001).

7. P. Wegrzyn, "Parametric resonance in a vibrating cavity," Physics Letters A, Vol.322, p.263-269 (2004).

8. A.A. Popov, "Parametric resonance in cylindrical shells: a case study in the nonlinear vibration of structural shells,” Engineering Structures, Vol.25, p.789-799 (2003).

9. M-F. Yu, G. J. Wagner, R. S. Ruoff, M. J. Dyer, "Realization of parametric resonances in a nanowire mechanical system with nanomanipulation inside a scanning electron microscope,” Physical Review B, Vol.66, (2002).

10. C. Ataman, H. Urey, “Nonlinear frequency response of comb-driven microscanners,” MOEMS Display and Imaging Systems II, Proc. SPIE Vol. 5348 (2004).

11. H. Schenk, P. Dür, D. Kunze, H. Lakner, H. Kück, “A resonantly excited 2D micro-scanning-mirror with large deflection," Sensors and Actuators A, Vol. 89, p.104-111 (2001).

12. H. Shenk, P. Dür, T. Haase, D. Kunze, U. Sobe, H. Lakner, H. Kück, "Large deflection micromechanical scanning mirrors for linear scans and pattern generation," Journal of Selected Topics in Quantum Electronics, Vol. 6, No. 5, p.715-722 (2000).

13. H. Schenk, A. Wolter, H. Lakner, "Design optimization of an electrostatically driven micro scanning mirror," Conf. on MOEMS and Miniaturized Systems, Proc. SPIE, Vol. 4561, p.35-44, San Francisco, Calif., USA, (2001)

14. A. H. Nayfeh, D. T. Mook, “Nonlinear Vibrations,” Jon Wiley and Sons, New York, (1995).

15. J.S. Rao, "Advanced Theory of Vibration," Jon Wiles and Sons, New York, (1992).

16. R. H. Rand, "Lecture Notes on Nonlinear Vibrations," Version 34a, Available online at http://www.tam.cornell.edu/randdocs, (2000).

17. K. L. Turner, S. A. Miller, P. G. Hartwell, N. C. MacDonald, S. H. Strogatz, "Five parametric resonances in a microelectromechanical system,” Nature, Vol. 396, p.149-152 (1998).

18. J. Chen, S. Kang, “Dynamic macromodeling of MEMS mirror devices, ” International Electron Device Meeting, Proc. IEEE, p.41.5.1-41.5.4, Washington DC, USA, (2001). 